\title{
POLÍTICAS DE POBLACIÓN Y DESARROLLO PARA EL AÑO 2000*
}

\author{
LuIS Olivos
}

\begin{abstract}
No es probable que comenzando el año 2000 los poetas encabecen una sublevación mundial para que se reparta la poesía. La poesia se repartirá como consecuencia del progreso humano, del desarrollo y del acceso de los pueblos al libro y a la cultura.
\end{abstract}

Pablo Neruda, 1968

\section{INTRODUCCIÓN}

ADEMÁS DE LOS VALORES cuantitativos proyectados por las instituciones internacionales -Naciones Unidas, CELADE, CEPAL, etc- que estiman el nivel de la población que ocuparía la región latinoamericana, existen ciertas implicaciones o posibles alternativas socioeconómicas y políticas con las cuales podríamos prever posibles "escenarios" futuros acerca de la situación de nuestro conjunto de países en el año 2000. Sin embargo, lo que debe preocuparnos es el tipo de sociedad a desarrollar y la responsabilidad de crear los instrumentos eficaces para lograr el indispensable cambio social y económico en los próximos años.

Las cifras elaboradas tienen validez como medida de la realidad próxima y como tal debemos aceptarlas. Las causas "naturales" de disminución de la población (desastres, guerras, calamidades) resultan tan imnnnderables como la adopción masiva de campañas oficiales controladoras de la natalidad en los diferentes países. Se deben considerar, entonces, las necesidades de la población latinoamericana en el año 2000 y las medidas indispensables para satłfacerlas, saliéndose imaginativamente de los marcos tradicionales de la planificación actual del desarrollo; aun más, se debe pensar en la necesidad de forzar la quiebra de los factores que impiden la planificación dentro de las estructuràs existentes.

Los objetivos de este trabajo son:

1. Presentar datos descriptivos de requerimientos en algunos sectores sociales en el año 2000 según las proyecciones establecidas.

* Este trabajo fue presentado originalmente al VII Congreso Interamericano de Planificación, celebrado en Lima del 20 al 25 de octubre de 1968. Aparecerá en inglés en Economic Development and Cultural Change. El autor es subdirector auxiliar del Departamento de Asuntos Sociales y jefe del Programa de Población. Las opiniones expresádas en el documento son las del autor y no reflejan necesariamente los puntos de vista de la Secretaría General de la OEA. 


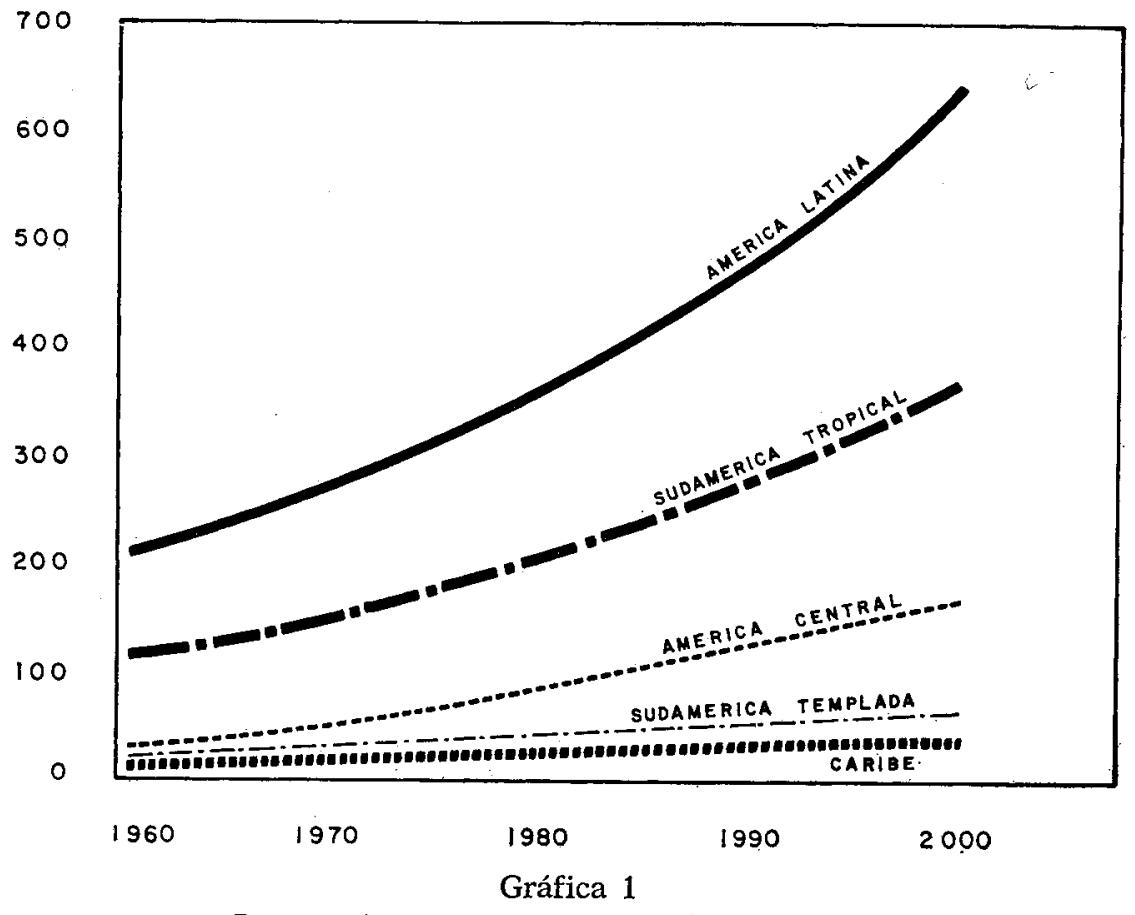

ProyeCción de La pOBLACí́N de AMÉrica LatiNa

2. Reconocer que la planificación puede ser una herramienta eficaz para analizar, guiar y promover las reformas estructurales e institucionales necesarias para el proceso de desarrollo latinoamericano, señalando que, sin embargo, se nota una crisis y una distorsión en sus funciones, especialmente en la no consideración equilibrada de los aspectos sustantivos del cambio social. Cabe mencionar, a este respecto, que resulta indispensable que se elabore una metodología que permita el ajuste de la planificación económica y de la social, por ejemplo, en cuanto a plazos, más allá de los términos acostumbrados, en que aun el largo plazo y las perspectivas resultan insuficientes en las consideraciones reales del próximo desarrollo.

3. Señalar que, si se desea satisfacer las necesidades y justas aspiraciones de los pueblos, es necesario revisar y reformular los planteamientos tradicionales de la planificación del desarrollo, en vista de las tendencias demográficas en América Latina y los obstáculos que se presenten. Simultáneamente a los cambios a fondo en las estructuras socioeconómicas de nuestros países -así como los cambios en las relaciones entre cada uno de ellos y con otros países del mundodeben llevarse a cabo políticas de población que sean "el conjunto coherente de decisiones que conforman una estrategia racional adoptada por el sector público, de acuerdo a las necesidades y aspiraciones de la colectividad, para desarrollar, conservar y utilizar los recursos humanos influyendo sobre la magnitud y el crecimiento probables de 
la población, su distribución por edades, la constitución y composición de las familias, la localización regional o rural-urbana de los habitantes, y la incorporación a la fuerza de trabajo y a la educación, con el fin de facilitar los objetivos del crecimiento económico y posibilitar la participación de la población en las responsabilidades y beneficios del progreso". 1

4. Señalar que el crecimiento de la población estimado para el año 2000 en América Latina debe ser encarado por los planificadores, por los técnicos, por los grupos dirigentes, por la sociedad toda, no como un puro ejercicio académico, sino como un desafío presente, ante el cual debe elaborarse una planificación con sentido regional, que reconozca las características de nuestros países y sus objetivos últimos, y que sea dada no solamente por la cantidad de individuos que pueda calcularse, sino por la calidad de la vida humana que se quiere alcanzar.

II. El CRECIMIENTO DE LA POBLACIÓN Y LAS NECESIDADES SOCIALES EN AmÉrica Latina

En 1920 había 90 millones de personas en América Latina y en 1960 la población alcanzó a 212 millones. Es decir, para el año 1960 la población era casi dos veces y media lo que había sido 40 años antes. De acuerdo con los cálculos hechos, en los próximos doce años, en 1980, seremos casi 380 millones en nuestra región. En el año 2000 llegaremos a los 640 millones si continúan las tasas actuales de nacimiento y mortalidad (véase el cuadro 1 ).

\section{Cuadro 1}

Proyecciones de la poblactón de América Latina (Millones)

\begin{tabular}{lrrrrr}
\hline & 1960 & 1970 & 1980 & 1990 & 2000 \\
\hline América Latina & 212 & 284 & 378 & 498 & 639 \\
Sudamérica Tropical & 112 & 154 & 210 & 280 & 362 \\
Centroamérica & 47 & 65 & 90 & 125 & 166 \\
Sudamérica Templada & 33 & 39 & 46 & 53 & 61 \\
Caribe & 20 & 26 & 32 & 40 & 50 \\
\hline
\end{tabular}

Fuente: Naciones Unidas, World Population Prospects, Nueva York, 1966, p. 134. (Doc. ST/SOA/Ser.A/41.)

El proceso de crecimiento de la población debido a una mortalidad decreciente y a una fecundidad constante ha existido por muchos años en la mayoría de los países latinoamericanos y podría seguir existiendo. Sin duda la región puede absorber millones de personas más en los 32 años que restan para el año 2000 , pero eso no significa que nin-

1 Unión Panamericana, Reunión sobre Políticas de Población en Relación al Desarrollo en América Latina (Caracas, 11-16 de septiembre de 1967), Informe Final, Washington, D. C., 1967. (Doc. UP/Ser.H/V/REPO/II/17). 
gún gobierno u organización internacional pueda mirar con tranquilidad esa perspectiva. El problema es determinar cuáles serán los cambios para orientar este crecimiento, cuándo se iniciarán con la intensidad necesaria para que tengan efecto y quiénes asumirán la responsabilidad de promoverlos en los países. Esto sin considerar la "trampa poblacional" a que se refiere Herman Kahn," en que los aumentos del ingreso per capita tienden a ser acompañados por nuevas actividades en salud pública que traen como efecto una disminución en las tasas de mortalidad, obteniéndose aumentos adicionales de población de forma que los aumentos per capita son anulados.

Quizás, para iniciar la consideración de problemas específicos, vale la pena referirse primero al desarrollo urbano. Las cifras que se presentan en el cuadro 2 son significativas. Las tendencias de una población rural que mantuviera los niveles actuales de emigración hacia la ciudad tendría repercusiones incalculables, tanto en los aspectos económicos y sociales como en los culturales y políticos. En este último sentido se piensa que una aglomeración urbana (se habla de una "ruralización de las ciudades") produciría, apreciándolo en forma positiva, una mayor "participación social" y la consiguiente democratización de los países; en lo negativo, mayores tensiones sociales debido a la incápacidad de los gobiernos para proporcionar servicios a una población donde la emergencia de nuevos y mayores problemas se hace cada vez más evidente.

\section{Cuadro 2}

Proyecciones de la poblactón urbana y ruRal de América latina (Porcientos)

\begin{tabular}{rrrrr}
\hline & 1950 & 1962 & 1980 & 2000 \\
\hline Población urbana & 39.0 & 47.1 & 59.4 & 73.0 \\
Población rural & 61.0 & 52.9 & 40.6 & 27.0 \\
Total & 100.0 & 100.0 & 100.0 & 100.0 \\
\hline
\end{tabular}

Fuente (datos de 1950 y 1962): Comisión Económica para América Latina, Estudio Económico de América Latina 1964, Nueva York, Naciones Unidas.

Los problemas de empleo, de una justa distribución del ingreso, de mayores oportunidades de participación y movilidad social, de la adecuación del medio físico, todos ellos agregados a un crecimiento continuo de los nuevos grupos sociales marginales, tienen que considerarse integrados en la planificación del desarrollo para los próximos 32 años, antes que su complejidad haga más difícil y costosa su consideración.

Una de las formas de hacer gráfico el impacto de la población sobre el desarrollo es anotar algunas de las implicaciones de la estructura por edades en la distribución de inversiones para bienestar social. Actualmente la población latinoamericana es joven y la expectativa más

2 Herman Kahn, "Economics", en Foreign Policy Association, Toward the Year 2018, Nueva York, Cowles Education Corporation, 1968. 
probable para los próximos 30 años es similar, existiendo alrededor del $40 \%$ menor de 15 años y sólo $3 \%$ mayor de 65 . De esta distribución por edades se desprende que en el área de la salud deben dedicarse importantes sumas para mantener programas de atención materno-infantil y en el área de la educación los aportes principales deben estar dirigidos al sector de la enseñanza primaria. Si observamos el caso de Suecia, a modo de comparación, cuya población menor de 15 años es del $20 \%$ y la mayor de 65 es de un $13 \%$, encontraremos que sus inversiones en educación están distribuidas equilibradamente entre la enseñanza a niveles primario, secundario y universitario, lo que permite obtener recursos humanos altamente capacitados. En cuanto al área de la salud, atención preferencial es dada a la geriatría, a los asilos $y$, en fin, a todos los sistemas de seguridad social destinados a servir a la población vieja.

Un alto porciento de población joven tiene repercusiones amplias en la familia, en la economía y en la sociedad toda. En México, en Centroamérica, en la parte tropical de América del Sur, hay alrededor de 960 personas en edad dependiente por cada mil en edad económicamente activa de 15 a 64 años. Pero aun así, esta cifra debe analizarse teniendo en cuenta que la mayoría de las mujeres en esas edades no tiene empleo productivo, estando dedicadas al cuidado del hogar y de los niños. Si las mujeres, los mayores de 65 años y los niños menores de 15 años se consideran dependientes de la población masculina cuyas edades fluctúan entre los 15 y los 64 años, existen alrededor de 2500 dependientes por cada 1000 hombres de edades entre $15 \mathrm{y}$ 64 años.

Con el fin de no insistir demasiado entre esta relación de los componentes sociales del desarrollo con el crecimiento acelerado de la población, en particular con aspectos tan evidentes como las necesidades de vivienda, nos referiremos al sector educación. Es éste el más estudiado y definido de todos los sectores. Políticas, planes y programas educacionales han sido elaborados a todos los niveles. Junto con el sector salud, el de educación tiene la ventaja de ser mayormente orientado, dirigido y administrado por cada gobierno. Existen datos estadísticos en casi todos los países para evaluar la eficiencia de los sistemas educacionales: relaciones de matrícula por grupo; asistencia escolar; número de alumnos por profesor, etc. Estos datos permiten la elaboración de planes y programas y el cálculo preciso de su financiamiento, y son elaborados por las propias oficinas nacionales de planificación educacional y complementados por los esfuerzos censales.

Naciones Unidas publicó en 1964 un estudio con el fin de ayudar a los gobiernos a utilizar los resultados de los censos en los trabajos de planificación económica y social.3 En el capítulo VII, "Estudios relativos a la situación y los problemas de la enseñanza", se señala: "Los estudios basados en los resultados de los censos de población y otras estadísticas demográficas constituyen, iunto con las estadísticas sobre la matrícula escolar y otras actividades de las instituciones

3 Naciones Unidas, Departamento de Asuntos Económicos y Sociales, Utilización de los programas nacionales de análisis de los datos de los censos de población en la formulación de planes y politicas, Nueva York, 1964. (Estudios sobre Población, Núm. 36.) 
docentes, un medio de evaluar la situación y el progreso de un país en lo referente a la enseñanza, la eficacia de su sistema docente, las necesidades en la materia y las inversiones necesarias para la construcción de escuelas, la formación de maestros, etc. ..." Creo que merecen subrayarse estos estudios dada la próxima realización del Programa de Censos de 1970 cuyo capítulo referente a enseñanza es extenso y deberá ser utilizado provechosamente en la planificación educacional para el año 2000.

Por razones conocidas la planificación del sector educación en nuestros países no es satisfactoria.4 Sin entrar en los aspectos de contenido, esto es, de "educación para el desarrollo", nos referiremos, de acuerdo al tema que se nos ha asignado, a la expansión del sistema educacional, tanto por motivos de la población creciente como por los esfuerzos

1950

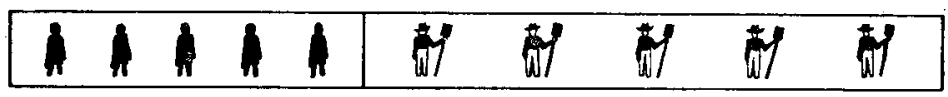

1962

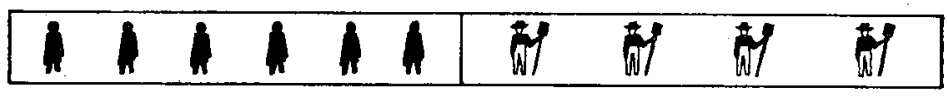

1980

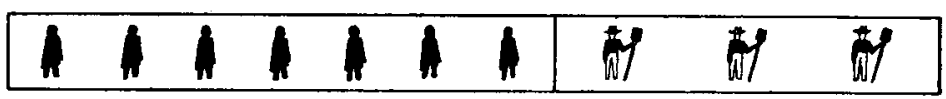

2000

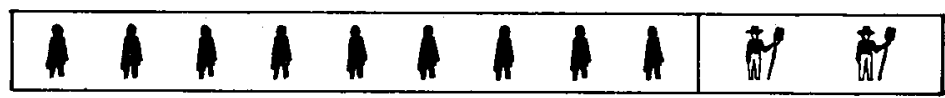

$\%$

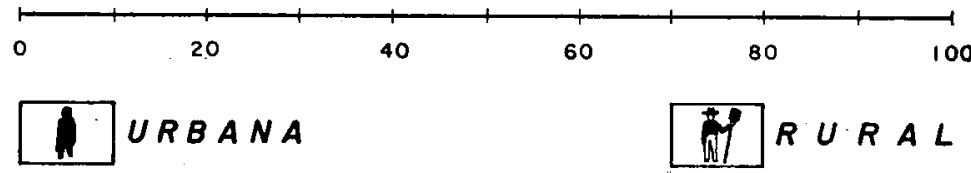

Gráfica 2

PROYección de la población URBaNa y RURAL de América Latina

de los países para el desarrollo de los recursos humanos. Todos nuestros países contemplan la educación primaria obligatoria con una duración y calidad que permitan al egresado poder cumplir con sus derechos y obligaciones de ciudadano y como productor y consumidor en una economía moderna. Fuera de la verdadera eficacia práctica de este planteamiento, la mayoría de los países aboga por una prolongación gradual de la educación, tanto más cuanto mayor sea la capacidad del Estado para financiar sus costos y las necesidades correspondientes de desarrollo. A este respecto cabe mencionar el caso de Chile que

4 A este respecto ver en UNESCO, Problems and Strategies of Educational Ptanning, París, 1965, los trabajos de Máximo Halty Carrere, Sylvain Lourié y Marshall Wolfe. 
expandió el año pasado su educación primaria obligatoria de seis a ocho años. A su vez estamos siendo testigos de las crecientes demandas de ingreso a las universidades en varios países.

Volvamos al crecimiento de la población y al año 2000. Los niños entre 5 y 14 años deben ser considerados como estudiantes primarios, o por lo menos deberían estar en la escuela primaria obligatoria. Los niños de estas edades serán alrededor de un $59 \%$ más numerosos en 1975 que en 1960. En el año 2000 serán tres veces más numerosos $(190 \%)$. Esto sin extenderse sobre las necesidades de construcciones escolares, maestros, administración, etc., ya que es evidente que la inversión en infraestructura, incluyendo recursos humanos, es de tal magnitud que se hace necesario buscar soluciones desde ahora.

En 1975 los adolescentes entre 15 y 19 años aumentarán en un $57 \%$ más que en 1960 , y en el 2000 serán más de tres veces más numerosos $(222 \%)$. Creemos necesario señalar la importancia de tener en cuenta en la planificación social este segmento de la población, con el fin de motivarlo y adaptarlo mentalmente a los nuevos valores y para impulsar su participación social.

\section{El DESafío al PLANIFICAdOR Pára EL aÑo 2000}

Las cifras presentadas en la sección anterior son reales porque responden a un crecimiento presente debido al decrecimiento de la mortalidad y a las tasas constantes de fecundidad. Como ya se ha dicho, y dentro del contexto de diferentes culturas, la mortalidad no puede ser aumentada; la fecundidad en cambio puede ser modificada. Los problemas son, como lo señaláramos anteriormente, cuándo, por quién y cómo, ya que el para qué desarrollista creemos que es conocido.

La contribución del control de la natalidad a los esfuerzos para el desarrollo han sido probados por destacados economistas y los argumentos en contra han sido rebatidos. Creemos que la discusión ya es parte de la temática diaria a favor o en contra del control de la natalidad y son comunes argumentos tales como:

1. "El crecimiento de la población no es problema porque nuestro país puede llegar a tener $x$ cantidad de población." El problema no es la cantidad de población que pueda llegar a tener un determinado país sino que el plazo para tenerla es demasiado corto, debido al crecimiento acelerado de su población mayor a sus posibilidades de crecimiento económico.

Jones ${ }^{5}$ ha estimado la interrelación entre las dos variables:

\begin{tabular}{|c|c|c|}
\hline $\begin{array}{l}\text { Si la población } \\
\text { crece al año el }\end{array}$ & $\begin{array}{l}\text { El pais ha de ahorrar e in- } \\
\text { vertir cada año al menos el }\end{array}$ & Tan sólo para \\
\hline $\begin{array}{l}1 \% \\
2 \% \\
3 \% \\
2 \% \\
3 \%\end{array}$ & $\begin{array}{r}\left.\begin{array}{l}3 \% \text { del ingreso nacional } \\
6 \% \text { del ingreso nacional } \\
9 \% \text { del ingreso nacional }\end{array}\right\} \\
9 \% \text { del ingreso nacional } \\
15 \% \text { del ingreso nacional }\end{array}$ & $\begin{array}{c}\text { Mantener estable el ingreso por } \\
\text { habitante, o sea, sin mejorar } \\
\text { los niveles medios de vida } \\
\text { lograr un aumento anual de } 1 \% \\
\text { lograr un aumento anual de } 2 \%\end{array}$ \\
\hline
\end{tabular}


Esta relación entre el ritmo de crecimiento de la población y el monto de los ahorros e inversiones que debe hacer el país describe claramente los esfuerzos para $a$ ) mantener las condiciones económicas actuales sin mejorar el ingreso anual medio por habitante y $b$ ) lograr un aumento del ingreso per capita.

2. "Nuestro país no tiene problemas demográficos porque tenemos todo el interior prácticamente deshabitado y una mayor población ayudaría a poblarlo." Típica respuesta nacionalista - junto a la de "una mayor población hace un país más poderoso"- que no toma en cuenta las necesidades de inversión en infraestructura que significaría adecuar esas regiones. ${ }^{6}$

3. "A medida que aumentan la industrialización y urbanización -mayores niveles educacionales - las tasas de fecundidad disminuirían en la misma forma que ha sucedido en Europa." El ejemplo no es comparable, porque tomó a los países europeos alrededor de 60 ó 70 años disminuir sus tasas de nacimiento, a pesar de los niveles culturales existentes. Los nacimientos en nuestros países alcanzan a 40 por 1000 ; para disminuir esta tasa a normas europeas (17 a 20 por 1000) debería obtenerse una reducción anual de siete millones de nacimientos. ${ }^{7}$

Creo que éstos son algunos de los puntos de discusión más importantes. Es aventurado entrar en este momento (octubre de 1968) en los aspectos culturales (incluyendo religiosos) de la planificación familiar después de la encíclica De la Transmisión de la Vida. Todas las incontables encuestas hechas entre mujeres católicas pre-encíclica deberán ser repetidas y en plazos diferentes. Debemos señalar que una encuesta realizada diez días después de la encíclica en una clínica de planificación familiar en Centroamérica dio como resultados que más del $60 \%$ de las mujeres católicas encuestadas dijeron que "no sabían nada de eso". Esto da mucho para pensar acerca de la sabiduría "avispada" de nuestras mujeres y su conducta futura. Tampoco creo que - debamos entrar en discusión con aquellos que dicen que el control de la natalidad es una conspiración entre los Estados Unidos y las oligarquías de nuestros países para limitar el aumento de las clases de bajos ingresos que son las que tienen más hijos y las que se consideran son el peso mayor sobre los gobiernos y la ayuda externa, y el verdadero caldo revolucionario. ${ }^{8}$ Menos creo que vale la pena discutir que una mayor población significa un mayor mercado, ni que la adaptación al medio de ciertas especies sirve de ejemplo a un equilibrio poblacional como en el caso de las moscas, que a pesar de su enorme poder reproductivo, todavía no logran cubrir la Tierra.

Nótese que hasta aquí hemos hablado de control de la natalidad y

Center for International Economic Growth, 1962. Citado en Problemas de población en relación con el desarrollo en América Latina, Unión Panamericana, Washington, D. C., 1965. (Doc. UP/Ser.H/VII.35.)

6 Ver, para Brasil, Rubens Vaz da Costa, Necesidad de una politica de población como parte de la politica de desarrollo, Unión Panamericana, Washington, D. C., 1968 (Doc. UP/Ser.H/VII.56.)

7 J. Mayone Stycos, "The Outlook for World Population", Science, Vol. 146, Núm. 3650, diciembre 11, 1964, pp. 1435-1440.

8 Aníbal Pinto (CEPAL) en el documento Sobre crecimiento demográfico y desarrollo (sin publicar) descartaba el eufemismo del término explosión demográfica diciendo que existe una "explosión de los pobres". 
no del concepto general de políticas de población que puede o no incluir un control de la población dependiendo de las características y necesidades de cada país. Y aquí insistimos en la definición de políticas de población que señaláramos al iniciar este trabajo. A este respecto cabe señalar que para muchos políticas de población y aun demografía sólo significan control de la natalidad o planificación familiar; habrá que aclarar todos estos términos ya que nos vamos encontrando con instituciones de estudios demográficos cuyo objetivo principal es distribuir contraceptivos.

Si sólo pensáramos en disminuir las tasas de nacimientos podríamos proponer, junto al suministro de medios e información sobre planificación familiar, la siguiente lista de "horrores" como las llama Kingsley Davis: ${ }^{9}$ "apretar a los consumidores mediante impuestos e inflación; limitar la construcción para hacer escasas las viviendas; forzar a las esposas y a las madres a trabajar fuera del hogar para contrarrestar la insuficiencia de los salarios masculinos, pero proveyendo pocos medios para el cuidado de los niños; estimular la migración a las ciudades pagando bajos salarios en el campo y proveyendo pocos empleos rurales; aumentar la congestión en las ciudades por inanición del sistema de tránsito; aumentar la inseguridad personal fortaleciendo las condiciones que producen desempleo y con arrestos políticos al azar". Como bien dice Kingsley Davis, no habría gobierno dispuesto a reducir sus tasas de nacimientos por medio de estas privaciones.

El profesor Davis dice también en el mismo artículo que los gobiernos cuentan con dos instrumentos poderosos: su dominio sobre la planificación económica y su autoridad (real o posible) sobre la educación. Mediante el primero se determinan - hasta donde puede hacerlo una política- las condiciones económicas y las circunstancias que afectan la vida de todos los ciudadanos; el segundo proporciona los conocimientos y actitudes necesarios para poner en ejecución los planes. El sistema económico, sigue diciendo, determina mayormente quién trabaja, qué puede comprarse, cuánto cuesta criar los hijos y cuánto puede gastar la persona. Las escuelas definen las funciones de la familia y desarrollan intereses vocacionales y de recreo; podrían, si se desease, definir de nuevo el papel de los sexos, desarrollar intereses que trasciendan el hogar y transmitir conocimientos realistas (en lugar de puramente moralistas) sobre el matrimonio, la conducta sexual y los problemas de población. Cuando el asunto se examine con ese criterio, termina diciendo en este párrafo, es evidente que los ministerios de economía y de educación, y no los de salud, deberían estar a cargo de las políticas de población.

Volvemos entonces al tema central de nuestro trabajo. Las políticas de población deben estar integradas a las políticas generales de desarrollo de nuestros países porque, de acuerdo a lo señalado en la Reunión de Caracas, ${ }^{10}$ por una parte, contribuyen a la eficacia de la

O Kingsley Davis, "Population Policy: Will Current Programs Succeed?", Science, Vol. 158, Núm. 3802, 10 de noviembre de 1967; traducido como "Política de población : ¿tendrán éxito los programas actuales?", DEMOGRAFía Y ECONOMía, Vol. III, Núm. 2, 1969, pp. 201-229.

yo Ver Unión Panamericana, op. cit. 
política general de desarrollo económico y social y, por otra, procuran que la familia, como sujeto y objeto del desarrollo, se desenvuelva en las mejores condiciones posibles de bienestar material y espiritual, respetando, en lo que hace al tamaño de la familia, el derecho de la pareja conyugal para ejercer su libertad mediante la información y los medios adecuados. El crecimiento de la población significa un desafío para los planificadores que debieran considerarlo el común denominador entre la planificación económica y la planificación social.

La impartancia del papel que puede jugar el análisis demográfico para establecer un enlace entre la realidad presente y las metas de planificación a largo plazo, se torna evidente si se considera que los niños que ahora están entrando a la escuela primaria son los mismos individuos que a comienzos del próximo siglo estarán a mitad de camino de su participación en la fuerza de trabajo de nuestros países. De ahí que lo que puedan transmitir hoy las aulas determinará, en gran medida, la composición educacional de la fuerza de trabajo y su nivel de productividad para el año 2000. La eficacia del sistema educativo actual -quizá más que cualquier otro factor- moldeará también el estilo de vida familiar futuro, en la medida en que los estudiantes de hoy estén mejor dotados para sus papeles como cabezas de familia $y$, sin duda, afectará la calidad de la sociedad del mañana al capacitarlos para ser ciudadanos más responsables.

Esta sucesión en cadena de generaciones que vibra a través de la trama de cualquier sociedad constituye para el planificador el medio operativo más eficaz para alterar el curso del crecimiento socioeconómico futuro. La base de recursos humanos con que se cuenta y su desarrollo potencial es considerado el factor más importante, como piedra angular de la planificación a largo plazo, que lo que puede representar la disponibilidad de recursos o las perspectivas de acumulación de capital.

$\mathrm{Y}$ esto deja en las manos de quienes planifican la responsabilidad de orientar los destinos de nuestros países para el año 2000, fecha que nos resulta cercana y que, sin embargo, nos llega en un vacío metodológico. Verdaderamente nos preocupa esta responsabilidad. Más todavía porque conocemos algunas críticas justificadas al respecto. ${ }^{11}$ Originalmente se pensó que la planificación, entre otros fines, trazaría el sentido y los objetivos comunes de la acción del Estado para el desarrollo de los países a largo plazo. Sin embargo, obstáculos bien definidos están limitando esta tarea y transformando al planificador de larga visión y comprensión interdisciplinaria en técnico presupuestario destinado a la asignación de recursos sin criterios claros respecto a prioridades de desarrollo y envueltos en el trámite cotidiano. En el mejor de los casos, la planificación nacional se ha transformado en planificación regional dentro de los países como una manera de salvar las rigideces estructurales.

11 Ver ILPES, Discusiones sobre planificación, México, Siglo XXI Editores, S. A., 1966; Germánico Salgado, Los primeros esfuerzos de ptanificación en América Latina: Notas sobre una experiencia, Naciones Unidas (Doc. ISDP.3/A/ R.2), y La planificación del desarrollo en los países de menor desarrollo relativo đe América Latina, Naciones Unidas (Doc. ISDP.3/A/R.2/Add.), ambos documentos del Tercer Seminario Interregional sobre Planificación del Desarrollo, Santiago de Chile, 18-19 de mayo de 1968. 
Es por eso que creemos de tanta importancia referirnos al año 2000 y al problema de la población creciente. Como un llamado de alerta al planificador, en el cual creemos que existe una vocación de cambio social, reproducimos el cuadro 3 elaborado por Philip Hauser. ${ }^{12}$

\section{Cuadro 3}

POBLACión MUNDial, REgIONES MÁS DESARRolladas Y MENOS DESARROLladas, 1965, Y PROYECTADAS A LOS AÑOS 2000 Y 2018

(Estimación media del profesor Hauser)

\begin{tabular}{|c|c|c|c|c|c|c|}
\hline \multirow{2}{*}{ Area } & \multicolumn{3}{|c|}{$\begin{array}{c}\text { Población } \\
\text { (en millones) }\end{array}$} & \multicolumn{3}{|c|}{$\begin{array}{l}\text { Distribución } \\
\text { (porciento) }\end{array}$} \\
\hline & 1965 & 2000 & 2018 & 1965 & 2000 & 2018 \\
\hline Total mundial & 3295 & 6994 & 9710 & 100.00 & 100.00 & 100.00 \\
\hline Regiones más desarrolladas 1 & 1038 & 1574 & 1980 & 31.50 & 22.51 & 20.39 \\
\hline $\begin{array}{l}\text { Europa } \\
\text { Unión Soviética } \\
\text { América del Norte } \\
\text { Australia y Nueva Zelanda } \\
\text { Otras }\end{array}$ & $\begin{array}{r}445 \\
231 \\
214 \\
14 \\
134\end{array}$ & $\begin{array}{r}563 \\
403 \\
376 \\
26 \\
206\end{array}$ & $\begin{array}{r}657 \\
532 \\
496 \\
35 \\
260\end{array}$ & $\begin{array}{r}13.51 \\
7.01 \\
6.49 \\
0.42 \\
4.07\end{array}$ & $\begin{array}{l}8.05 \\
5.76 \\
5.38 \\
0.37 \\
2.95\end{array}$ & $\begin{array}{l}6.77 \\
5.48 \\
5.11 \\
0.36 \\
2.67\end{array}$ \\
\hline Regiones menos desarrolladas 2 & 2257 & 5420 & 7730 & 68.50 & 77.49 & 79.61 \\
\hline $\begin{array}{l}\text { Asia Oriental (excluyendo Japón) } \\
\text { Asia Meridional } \\
\text { Africa } \\
\text { América Latina (excluyendo las } \\
\text { zonas templadas de América } \\
\text { del Sur) } \\
\text { Otras }\end{array}$ & $\begin{array}{l}756 \\
978 \\
311\end{array}$ & $\begin{array}{r}1484 \\
2443 \\
864\end{array}$ & $\begin{array}{l}2016 \\
3515 \\
1266\end{array}$ & $\begin{array}{r}22.88 \\
29.68 \\
9.44\end{array}$ & $\begin{array}{l}21.22 \\
34.93 \\
12.35\end{array}$ & $\begin{array}{l}20.76 \\
36.20 \\
13.04\end{array}$ \\
\hline
\end{tabular}

Fuente: Las proyecciones hasta el año 2000 son las alternativas altas de las Naciones Unidas. Las estimaciones para 1965 son de las Naciones Unidas, Demographic Yearbook, 1966, cuadro 1.

1 Incluye Europa, Rusia, América del Norte, Japón, América del Sur templada, Australia y Nueva Zelanda.

2 Incluye Asia Oriental excluyendo Japón y Asia Meridional, Africa, América Latina y Oceanía excluyendo Australia y Nueva Zelanda.

El mismo Hauser en ese artículo resume el papel que puede tener la población en determinar los destinos políticos y económicos del mundo actual en los próximos años y señala los diversos aspectos que deben considerarse simultáneamente:

1. Vivimos en un mundo de naciones prósperas y de naciones no prósperas.

2. Las diferencias entre las naciones en sus niveles de vida se han transformado en diferencias "sentidas", debido a la "revolución de las expectativas crecientes". 
3. Las naciones no prósperas están luchando para alcanzar mayores niveles de vida y han hecho de este objetivo su mayor aspiración nacional (fuera del de independencia, para las que no lo han logrado).

4. Existe una correlación inversa entre los niveles de vida y las tasas presentes y proyectadas de crecimiento de la población.

5. El crecimiento rápido de la población está obstruyendo los esfuerzos para elevar los niveles de vida de las regiones en desarrollo.

6. A pesar de los esfuerzos nacionales e internacionales para elevar los niveles de vida, las diferencias entre las naciones prósperas y no prósperas están aumentando en vez de estar disminuyendo.

7. Las tasas aceleradas de urbanización en las regiones en desarrollo están aumentando las tensiones sociales, la inestabilidad política y pueden amenazar la paz mundial.

8. La agrupación política de un mundo bipolar - la confrontación entre naciones capitalistas o comunistas, o la guerra fría- está aumentando las tensiones que surgen de los esfuerzos frustrados para elevar los niveles de vida en las regiones en desarrollo.

9. El mundo político bipolar está siendo fragmentado por una división entre prósperos y no prósperos en ambos campos. Una posible reagrupación política puede tener como base, más que la actual división comunista-capitalista, una fundamentación próspero vs. no próspero. Esto produciría una confrontación Norte-Sur en vez de Oriente-Occidente. ${ }^{13}$

Estos nueve puntos del profesor Hauser nos plantean una situación real y cercana a la cual quisiéramos llevar a nuestros planificadores. Que así como se inició la planificación nacional hace algunos años y luego se han iniciado arreglos subregionales (ALALC, Centroamérica, Región Andina) debemos llegar a una verdadera integración latinoamericana planificada que tome en cuenta tanto sus aspectos formales como los sustantivos. Que para el año 2000 pensemos en nuestros países como un bloque regional digno y respetable en el concierto mundial. Que planifiquemos, más allá de las negociaciones comerciales y de la racionalización formal neutra del desarrollo económico, el tipo de sociedad que queremos alcanzar y los cambios indispensables para lograrla. 